\title{
Electrowetting on gold electrodes with microscopic three-dimensional structures for microfluidic devices
}

\author{
Hiroomi Yokomaku, Wataru Satoh, Junji Fukuda, and Hiroaki Suzuki ${ }^{\text {a) }}$ \\ Graduate School of Pure and Applied Sciences, University of Tsukuba, Tsukuba, Ibaraki, 305-8573, Japan
}

(Received 26 March 2008; accepted 8 July 2008; published online 22 September 2008)

\begin{abstract}
To improve the performance of electrowetting-based microfluidic devices, we used micropillar structures to enhance the changes in the wettability of gold electrodes. The changes in the contact angle of a sessile drop were influenced by the diameter of the micropillars and interpillar distances. For a potential change between $0 \mathrm{~V}$ and $-1.0 \mathrm{~V}$, the change of the contact angle of the $\mathrm{KCl}$ sessile drop was $41^{\circ}$ on a smooth electrode, but $88^{\circ}$ on an electrode with micropillars with a $10 \mu \mathrm{m}$ diameter. Furthermore, the existence of the micropillars accelerated the change of the contact angle. The gold electrodes with the micropillars were used to generate the capillary force to mobilize a liquid column in a microflow channel. Compared to a device with a smooth electrode, this device showed a fourfold increase in the flow velocity at $-0.9 \mathrm{~V}$. The electrodes were also used as a valve. The ability to stop an intruding solution and the switching speed was improved with the micropillar structure. (C) 2008 American Institute of Physics. [DOI: 10.1063/1.2976358]
\end{abstract}

\section{INTRODUCTION}

Electrowetting features a change in the wettability of a metal surface or a dielectric on an electrode in response to a potential change. ${ }^{1,2}$ The capability to change the surface state freely by an electrical signal has already opened up many possibilities for a variety of industrial applications. Among them, microfluidic transport is a critical application, and the technique has been actively incorporated in various forms in a trend of research relating to the micrototal analysis system or laboratory-on-a-chip. ${ }^{2-4}$ In microfluidic systems, most of the devices proposed earlier have mainly focused on the transport of droplets and were based on the so-called electrowetting-on-dielectric using an array of electrodes covered with an insulating layer. ${ }^{4-12}$ However, these systems required high voltage to control the interfacial tension. On the other hand, direct electrowetting on an exposed electrode surface can be controlled at an extremely low voltage $(\sim 1 \mathrm{~V}){ }^{6}$, which has made it possible to design a variety of functional microfluidic devices. ${ }^{13-15}$

A common principle for these devices is to change the wettability of a surface to mobilize a solution in a hydrophilic state and stop it in a hydrophobic state. In order to increase the flow velocity (hydrophilic state) and stop the transported solution at a desired location (hydrophobic state), however, a challenge has been to enhance the accompanying change. There are two critical factors that affect the wettability of a surface. The first is the surface free energy, which depends on the chemical nature of the material. The second is the surface morphology, many examples of which can be seen in nature, e.g., the papillae in the leaves of the plants. Various methods have been proposed for the construction of microscopic three-dimensional structures, particularly for the construction of superhydrophobic surfaces. These include the

\footnotetext{
a) Author to whom correspondence should be addressed. Tel.: +81-29-8535598. FAX: +81-29-853-4490. Electronic mail: hsuzuki@ims.tsukuba.ac.jp.
}

use of fractal structures, ${ }^{16}$ solvent evaporation, ${ }^{17}$ a sol-gel process, ${ }^{18}$ an oxygen plasma treatment, ${ }^{19}$ electrochemical deposition, ${ }^{20,21}$ seeded growth, ${ }^{22}$ deposition of microbeads, ${ }^{23}$ and plasma-enhanced chemical-vapor deposition. ${ }^{24,25}$ As a new approach, rough surfaces have also been realized with microfabricated structures. ${ }^{26-29}$ In addition, the control of the wettability of a rough surface by electrowetting has begun to be reported. ${ }^{30}$

In this study, we used microscopic three-dimensional structures to improve the performance of microfluidic devices based on electrowetting on a gold electrode. The characterization of gold electrodes with microscopic structures revealed that the change in the wettability was remarkably enhanced. The application of the electrode to the transport of continuous liquids showed accelerated transport and effective blocking of an intruding solution.

\section{EXPERIMENTAL}

\section{A. Reagents and materials}

Reagents and materials used for fabrication and characterization were obtained from the following commercial sources: glass wafers (No. 7740, 3 inch, $500 \mu \mathrm{m}$ thick) from Corning Japan (Tokyo, Japan); chemically amplified negative photoresist, TMMR S2000, negative photoresist, OMR83, thick-film positive photoresist, PMER P-LA900PM, from Tokyo Ohka Kogyo (Kanagawa, Japan); thick-film negative photoresist, SU-8, from MicroChem (Newton, MA); and precursor solution of polydimethylsiloxane (PDMS), KE-1300T, from Shin-Etsu Chemical (Tokyo, Japan). Other reagents of analytical reagent grades were obtained from Wako Pure Chemical Industries (Osaka, Japan). All solutions were prepared with distilled de-ionized water.

\section{B. Electrowetting on microscopic rough structures}

When a liquid droplet is placed on a smooth surface, the contact angle $\theta$ is given by Young's equation 


$$
\gamma_{\mathrm{SV}}=\gamma_{\mathrm{SL}}+\gamma_{\mathrm{LV}} \cos \theta
$$

where $\gamma_{\mathrm{SV}}, \gamma_{\mathrm{SL}}$, and $\gamma_{\mathrm{LV}}$ are the interfacial tensions at the solid-vapor, solid-liquid, and liquid-vapor interfaces, respectively. On a metal electrode, $\gamma_{\mathrm{SL}}$ can be changed by changing the potential of the electrode. When a simple HelmholtzPerrin parallel plate capacitor is assumed for the metalsolution interface, the Lippmann equation becomes ${ }^{31}$

$$
\gamma_{\mathrm{SL}}=\gamma_{0 \mathrm{SL}}-\frac{\varepsilon \varepsilon_{0}}{2 d} V^{2}
$$

where $\gamma_{0 S L}$ is a constant, $\varepsilon$ is the dielectric constant of the material in the capacitor, $\varepsilon_{0}$ is the permittivity of free space, $d$ is the interplate spacing, and $V$ is the voltage drop between the plates or the potential of the electrode measured with respect to the potential corresponding to the maximum $\gamma_{\mathrm{SL}}$.

When the surface is rough, the contact angle changes from $\theta$. Two representative models explain the observed changes. In the Wenzel model, ${ }^{32}$ the increase in the surface area is taken into account, and the observed contact angle is expressed as

$$
\cos \theta_{\mathrm{w}}=r \cos \theta
$$

where $r$ is the roughness factor defined as the ratio of the actual surface area to the projected surface area viewed from above. The Cassie-Baxter model assumes that the surface is made from different materials and the contact angle is expressed as follows: ${ }^{33}$

$$
\cos \theta_{\mathrm{C}}=f_{1} \cos \theta_{1}+f_{2} \cos \theta_{2} \text {. }
$$

In Eqs. (3) and (4), $\theta_{w}$ and $\theta_{c}$ represent the contact angle observed on the rough surface, respectively. In Eq. (4), $f_{1}$ and $f_{2}$ represent the area fractions of the different materials 1 and 2 and $\theta_{1}$ and $\theta_{2}$ represent the contact angles observed on surfaces consisting of only 1 and 2, respectively. When the composite surface consists of a solid surface and an air layer, Eq. (4) becomes ${ }^{26}$

$$
\cos \theta_{C}=f(\cos \theta+1)-1,
$$

where $f$ is the fraction of the solid area. The application of the models depends on the condition. In the following discussion, the contact angle on a rough surface will be collectively expressed as $\theta^{*}$.

\section{Fabrication of gold electrodes with microscopic three-dimensional structures}

Gold electrodes with micropillars were created on a PDMS substrate. First, arrays of microholes were formed on a glass substrate as a template with the thick-film positive photoresist (PMER P-LA900PM). The precursor solution of PDMS was then poured onto the template and cured. When the PDMS sheet was removed from the template, the patterns were transferred to the PDMS substrate as arrays of micropillars. The replicative process facilitated the preparation of many surfaces of the same microstructure. A gold layer was sputter deposited onto the PDMS substrate with a chromium intermediate layer [Fig. 1(a)]. As far as the experiment was concerned, the adhesion of the layer to PDMS was satisfactory. (a)

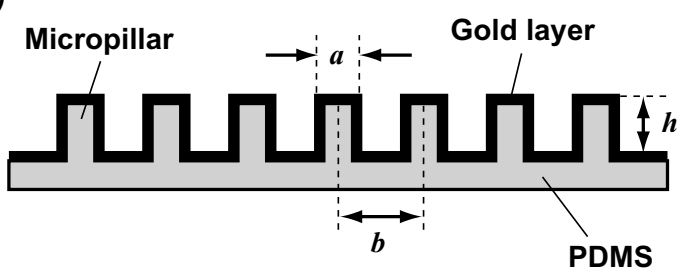

(b)

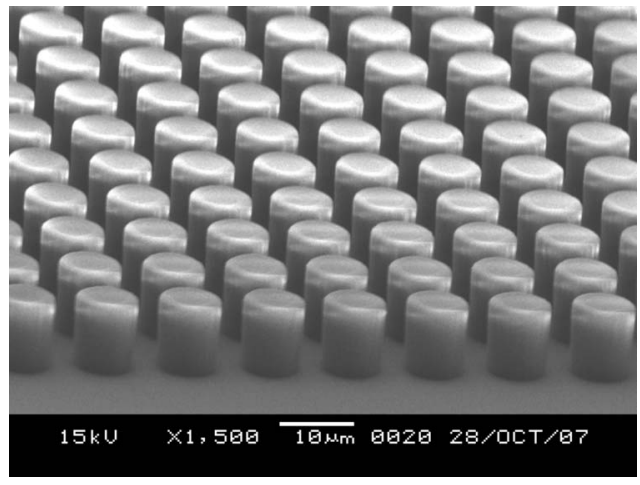

FIG. 1. Gold electrode formed on micropillars. (a) Cross section of the electrode showing related dimensions. (b) SEM photograph of micropillars $(a=10 \mu \mathrm{m}, f=0.45)$.

To measure the effect of the microscopic structures, pillar arrays of various dimensions were formed (Table I). In the following discussions, the diameter of the micropillars will be denoted as $a$, the interpillar distance (center to center) as $b$, and the height as $h$. In our experiments, $h$ was fixed at $10 \mu \mathrm{m}$. The roughness factor $r$ is expressed as

$$
r=1+\frac{\pi a h}{b^{2}}
$$

Similarly, $f$ is expressed as the fraction of the top area of the micropillars with respect to the entire projected area

$$
f=\frac{\pi a^{2}}{4 b^{2}} .
$$

\section{Structure and fabrication of microfluidic devices}

The effect of micropillars was also examined using the two types of microfluidic devices shown in Fig. 2. The structures were formed with a PDMS substrate with a flow channel structure and a glass substrate with electrodes. The flow channel structure was formed using patterns of a thick-film photoresist (SU-8) as a template. For the device shown in

TABLE I. Dimensions of the micropillars used in the experiments and corresponding values of $r$ and $f$.

\begin{tabular}{cccc}
\hline \hline $\begin{array}{c}a \\
(\mu \mathrm{m})\end{array}$ & $\begin{array}{c}b \\
(\mu \mathrm{m})\end{array}$ & $r$ & $f$ \\
\hline 10 & 13 & 2.8 & 0.45 \\
10 & 23 & 1.6 & 0.15 \\
20 & 26 & 1.9 & 0.45 \\
20 & 46 & 1.3 & 0.15 \\
50 & 66 & 1.4 & 0.45 \\
\hline
\end{tabular}




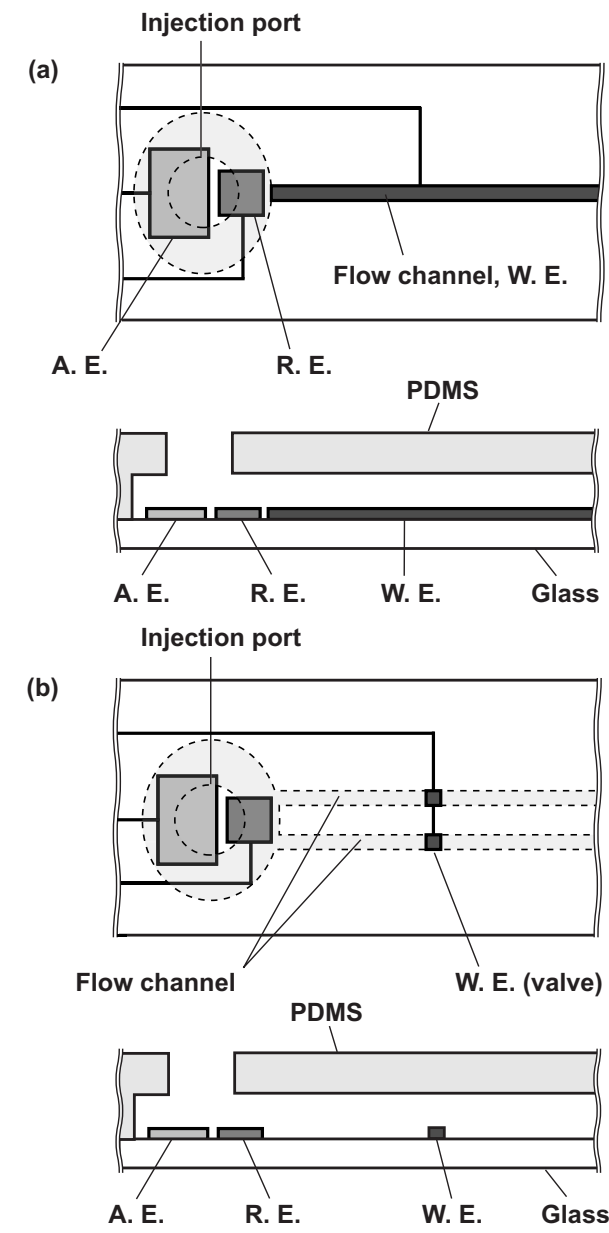

FIG. 2. Microfluidic devices used for evaluation. (a) Device used to measure the flow velocity. (b) Device used to check the valve action. In (a) and (b), the lower figures show cross sections along the flow channels. W. E., working electrode; R. E., reference electrode; A. E., auxiliary electrode.

Fig. 2(a), a long rectangular pattern of an array of micropillars was formed with the thick-film photoresist (TMMR S2000) only on the working electrode areas of the glass substrate [Fig. 1(b)]. After gold working electrodes were formed on the patterns of micropillars, a $\mathrm{Ag} / \mathrm{AgCl}$ reference electrode and a platinum auxiliary electrode were formed in the injection port. The negative photoresist (OMR-83) was used to insulate lead areas and form six pinholes on the $\mathrm{Ag} / \mathrm{AgCl}$ electrode. ${ }^{34}$ The $\mathrm{AgCl}$ layers were grown from the pinholes into the underlying silver layer by applying $1.0 \mu \mathrm{A}$ for 3 min with respect to a platinum electrode on the chip in a $1.0 \mathrm{M} \mathrm{KCl}$ solution whose $\mathrm{pH}$ was adjusted to 2.2 with $\mathrm{HCl}$ $\left(25^{\circ} \mathrm{C}\right)$. To avoid unwanted contamination of the working electrode surface, the active areas of the electrodes were delineated with the flow channel pattern of PDMS placed and sealed on the glass substrate. In all the complete devices, the height and width of the flow channel were 100 and $500 \mu \mathrm{m}$, respectively. For comparison, another device with a smooth working electrode was also formed. Components other than the working electrode and their dimensions were the same.

The structure with the gold electrode was also used as a valve [Fig. 2(b)]. A short working electrode was formed in a flow channel. The left edge of the electrode was located at 5 $\mathrm{mm}$ from the inlet of the flow channel. Another device with a smooth working electrode was also formed on the same chip in parallel. To apply the potential simultaneously, the two working electrodes were connected with a lead.

\section{E. Procedure for measurement of the change in the contact angle}

In measuring the contact angle under a constant applied potential, $5 \mu \mathrm{l}$ of a droplet of an electrolyte solution was carefully placed on a gold electrode using a microsyringe. A 1.0 M KCl solution was used unless otherwise noted. A thin $\mathrm{Ag} / \mathrm{AgCl}$ wire (diameter, $500 \mu \mathrm{m}$ ) was used as a reference electrode. The $\mathrm{Ag} / \mathrm{AgCl}$ electrode had been formed by growing a $\mathrm{AgCl}$ layer on a silver wire by applying $10 \mu \mathrm{A}$ for 20 min in a $1.0 \mathrm{M} \mathrm{KCl}$ solution, whose $\mathrm{pH}$ was adjusted to $\mathrm{pH}$ $2.2\left(25^{\circ} \mathrm{C}\right)$. The contact angle was first measured at $0 \mathrm{~V}$. The shape of the sessile drop was recorded using a Keyence VB-7010 charge coupled device (CCD) camera and a VBG25 multiangle measurement system. The contact angle was calculated from the video image. $\mathrm{The} \mathrm{Ag} / \mathrm{AgCl}$ electrode was then inserted into the sessile drop, and a negative potential was applied to the gold electrode with respect to the $\mathrm{Ag}$ / $\mathrm{AgCl}$ electrode using a potentiostat (HA-151, HokutoDenko, Japan). In measuring the contact angle, the reference electrode was temporarily removed from the sessile drop because the existence of the electrode deformed its shape. Since the three-phase boundary of the sessile drop was pinned, the shape of the sessile drop was fixed during the measurement. After the contact angle measurement, the Ag/ $\mathrm{AgCl}$ electrode was inserted again into the sessile drop. The same procedure was repeated until the potential reached $-1.0 \mathrm{~V}$. The experiments were carried out at room temperature.

\section{F. Principle of operation and procedure for microfluidic transport}

In examining the influence of the change in wettability on microfluidic transport, the two kinds of devices shown in Figs. 2(a) and 2(b) were used. In using the device shown in Fig. 2(a), a $1.0 \mathrm{M} \mathrm{KCl}$ solution containing fluorescein was filled in the injection port. When the potential of the long working electrode was $0 \mathrm{~V}$, the solution wetted only the edge of the electrode. However, when a negative potential was applied to the working electrode, the surface of the working electrode became more hydrophilic, and the solution was mobilized along the flow channel to the end of the electrode by a capillary action. Since the measurement of low flow velocities at a point in the flow channel was difficult, the flow velocity was calculated as the average between the edge at the entrance and at $10 \mathrm{~mm}$ from there.

In using the device shown in Fig. 2(b), a sample solution filled in the injection port was transported along the flow channel to the valve area by a capillary action because the surface of the glass substrate was hydrophilic. The intrusion of the solution into the valve area and the movement of the solution in the area were observed using the VB-G25 multiangle measurement system, the CCD detection system, and a fluorescent light source. The experiments were carried out at room temperature. 


\section{RESULTS AND DISCUSSION}

\section{A. Influence of the dimensional parameters of the micropillars on the change in wettability}

The state of the gold surface has been controversial. Immediately after the gold film is deposited, the surface is hydrophilic. However, once the film is exposed to the atmosphere, it rapidly becomes less hydrophilic. ${ }^{35,36}$ We were able to confirm that the contact angle was $29^{\circ}$ after it was taken out of the deposition chamber. However, the contact angle began to change rapidly; it reached $70^{\circ}$ and saturated at that angle after $30 \mathrm{~min}$. The change has been explained as the contamination by the carbonaceous compounds that exist in the atmosphere. Although the change of the surface state is unavoidable, the contact angle on a gold surface exposed for a sufficiently long time seems to be fairly reproducible in comparison to the previous results presented by the other groups. ${ }^{21,36,37}$

The roughness of the electrode surface is characterized by the variables in Eqs. (6) and (7). We first examined the effect of changing $a$ and $b$ with a fixed $f(0.45)$. Figure 3(a) shows the dependence of the contact angle on the applied potential obtained with three combinations of $a$ and $b$. Data obtained on a smooth electrode are also shown for comparison. With the largest micropillars $(a=50 \mu \mathrm{m})$, the change was slight compared to the smooth electrode. With the decrease in the pillar dimensions, the contact angle increased at more positive potentials but decreased at more negative potentials. With $a=10 \mu \mathrm{m}$, the change in the contact angle between 0 and $-1.0 \mathrm{~V}$ was $88^{\circ}$, whereas it was $41^{\circ}$ on a smooth electrode. The applicability of the Wenzel model and the Cassie-Baxter model is easily known by plotting $\cos \theta^{*}$ with respect to $\cos \theta$. Previous data in a related study came close to a straight line that passed the origin, ${ }^{38}$ suggesting that the change is explained well by the Wenzel model for $\theta \leq 90^{\circ}$. In our case, however, the data points were close to the lines anticipated from the Wenzel model in the sense that $\cos \theta^{*}$ is close to 1 but deviated from the lines at a substantially positive point [Fig. 3(b)]. The discrepancy was larger with the smaller micropillars. The results indicate that a hydrophilic surface becomes more hydrophobic, a tendency that is opposite to that in the Wenzel model. Similar tendencies have been observed with other surface structures and/or materials. $^{21,28}$ Abdelsalam et al. $^{21}$ reported the same tendency on a gold electrode with a nanostructure. Shirtcliffe $e t$ $a l{ }^{28}$ examined the change caused by changing the height or the aspect ratio of the micropillars and showed an increase in the contact angle from approximately $80^{\circ}$ to $>140^{\circ}$. On the other hand, even the point of the most hydrophobic state did not reach the theoretical line of the Cassie-Baxter model. These results indicate that the solutions partially wetted the side walls of the micropillars but did not drop onto the bottom of the structure in the changing region, leaving an air layer between the solution and the electrode.

We then examined changes observed when $f$ was changed with a fixed $a$ (Fig. 4). Here, electrodes with $f$ $=0.15$ and 0.45 were prepared with $a=10$ and $20 \mu \mathrm{m}$. When $a=20 \mu \mathrm{m}$, the difference was slight between the two cases of $f$. However, in the more positive potential region, the (a)
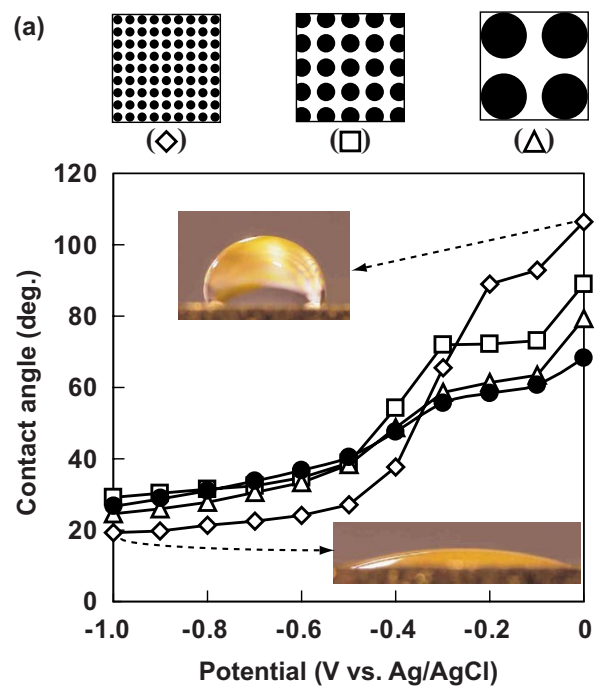

(b)

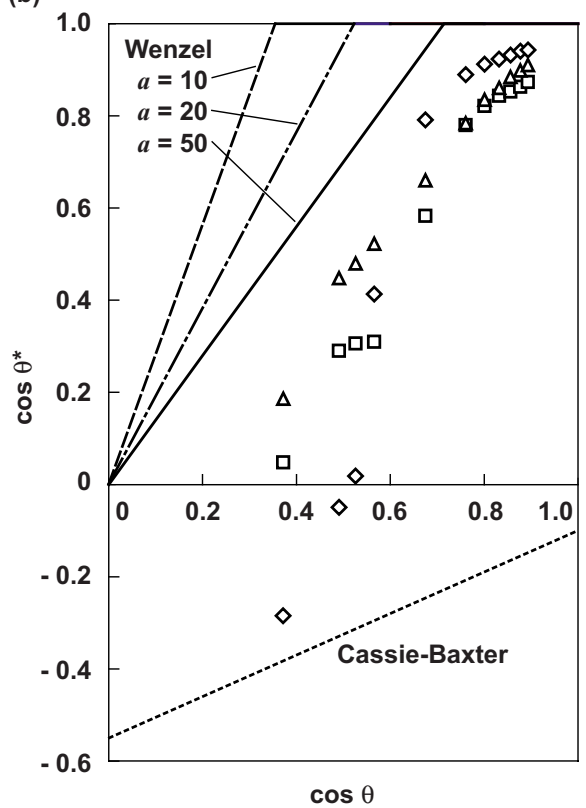

FIG. 3. (Color online) Effect of micropillars on electrowetting on gold electrodes. (a) Changes in the contact angle when $a$ was changed with a fixed $f(0.45)$. The upper figures show the relative size of the respective micropillar structures and interpillar distance for comparison. Three runs were made for each potential, and the averages were plotted. (b) Relationship between $\cos \theta^{*}$ and $\cos \theta$. The lines denoted as "Wenzel" and "CassieBaxter" indicate theoretical values anticipated from Wenzel's model and Cassie-Baxter's model, respectively, assuming that the models are applicable even in the indicated region. For the former, $a=10, a=20$, and $a$ $=50$ indicate that the lines correspond to the cases of pillar diameters of 10 , 20 , and $50 \mu \mathrm{m}$, respectively. Diameter of the pillars: $\diamond, 10 \mu \mathrm{m} ; \square$, $20 \mu \mathrm{m} ; \triangle, 50 \mu \mathrm{m}$. - shows the contact angle on a smooth electrode.

electrode with $f=0.45$ became more hydrophobic. When $a$ $=10 \mu \mathrm{m}$, a more enhanced change was observed with $f$ $=0.45$. Compared to the case of $f=0.15$, the electrode became more hydrophobic in the more positive region but more hydrophilic in the more negative potential region. Overall, surfaces with smaller $a$ and larger $f$ enhance the changes of wettability.

\section{B. Dependence of the contact angle on electrolytes}

So far, we have discussed the change in wettability of a gold surface when a droplet of $\mathrm{KCl}$ solution was used. How- 


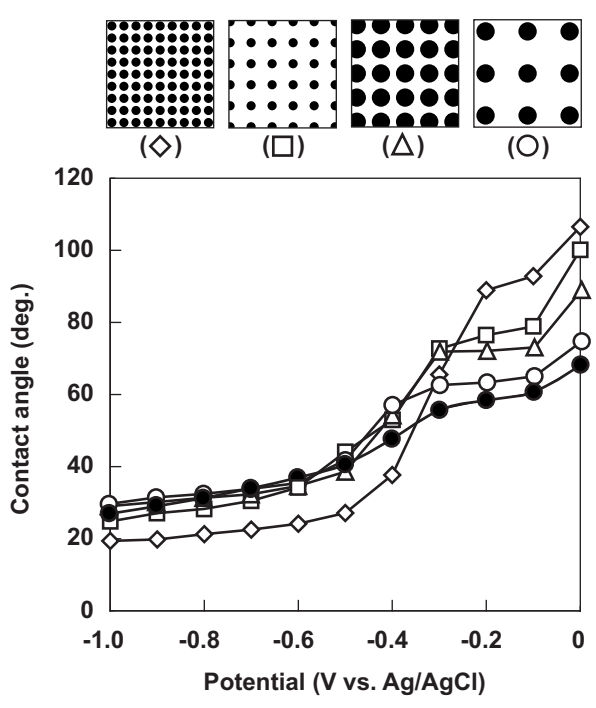

FIG. 4. Changes in the contact angle when $f$ was changed with two combinations of $a$. The upper figures show the relative size of the respective micropillar structures and interpillar distance for comparison. $\diamond, a$ $=10 \mu \mathrm{m}, f=0.45 ; \square, a=10 \mu \mathrm{m}, f=0.15 ; \Delta, a=20 \mu \mathrm{m}, f=0.45 ; \bigcirc, a$ $=20 \mu \mathrm{m}, f=0.15$. shows the contact angle on a smooth electrode. Three runs were made for each potential, and the averages were plotted.

ever, solutions with different ions may show different behaviors because of the difference in surface excess. ${ }^{31}$ Figure 5(a) shows changes in the contact angle when $1.0 \mathrm{M}$ solutions of $\mathrm{NaCl}, \mathrm{CaCl}_{2}$, and $\mathrm{MgCl}_{2}$ were placed on a smooth gold electrode. To stabilize the potential of the $\mathrm{Ag} / \mathrm{AgCl}$ reference electrode, chloride salts were used. For comparison, the change in the contact angle of a sessile drop of $\mathrm{KCl}$ solution is also shown. No significant difference was observed between the solutions of $\mathrm{KCl}$ and $\mathrm{NaCl}$. On the other hand, when the $\mathrm{CaCl}_{2}$ and $\mathrm{MgCl}_{2}$ solutions were used on a smooth surface, the changes were much smaller, although they were similar between the two solutions.

Contrary to the smooth electrode, the electrodes with micropillars showed remarkable enhancement in the changes [Fig. 5(b)]. The change in the contact angle between 0 and $-1.0 \mathrm{~V}$ increased to $74^{\circ}$ for $\mathrm{MgCl}_{2}$ and $77^{\circ}$ for $\mathrm{CaCl}_{2}$. Although such a change is still smaller than that of $\mathrm{KCl}\left(88^{\circ}\right)$, it was sufficient to cause the movement of the solution in the flow channel.

\section{Kinetic behavior of a droplet}

In Secs. III A and III B, we have focused on the changes between states. However, in applying this technique to microfluidic transport, the kinetic behavior of a droplet is also a concern. To examine how the microscopic three-dimensional structure affects the speed of the change in wettability, the shape of a sessile drop was recorded using a video camera after applying $-1.0 \mathrm{~V}$ (Fig. 6). When the change was caused on a smooth gold electrode, the contact angle changed by $90 \%$ spending about $2.5 \mathrm{~s}$ and almost settled at the final value in about $3 \mathrm{~s}$. On the other hand, on the gold electrodes with micropillars, the change was significantly accelerated, and, in faster cases, the $90 \%$ change between the two states occurred within a time that was less than the limit of resolution of the video camera $(<0.14 \mathrm{~s})$. (a)

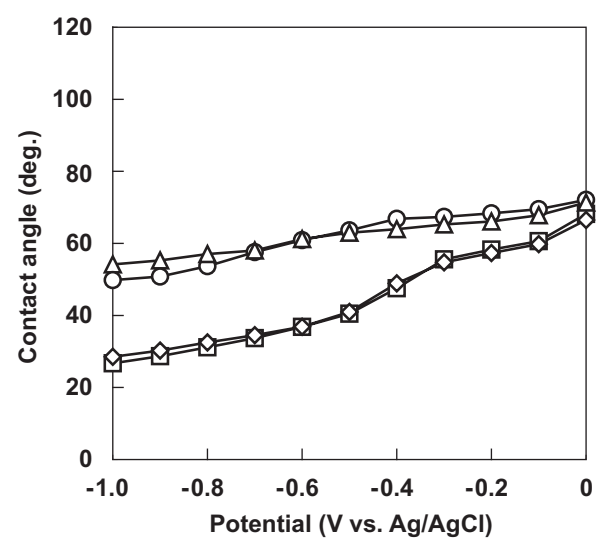

(b)

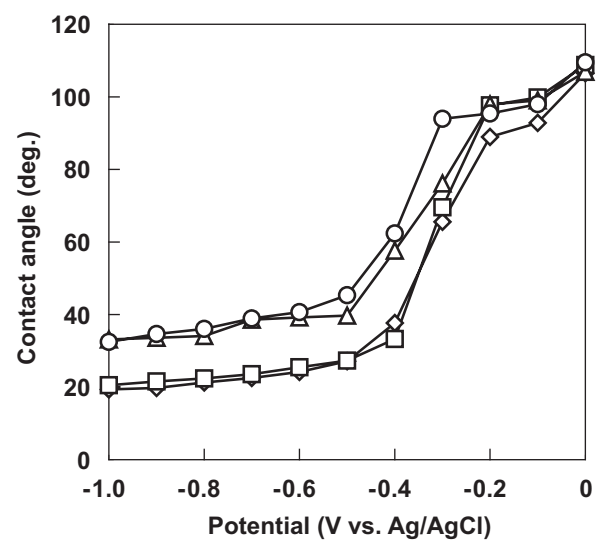

FIG. 5. Influence of electrolytes on the changes in the contact angle. (a) On a smooth electrode. (b) On electrodes with micropillars $(a=10 \mu \mathrm{m}, f$ $=0.45)$. Electrolytes: $\diamond, \mathrm{KCl} ; \square, \mathrm{NaCl} ; \triangle, \mathrm{MgCl}_{2} ; \bigcirc, \mathrm{CaCl}_{2}$. Concentration: 1.0 M. Three runs were made for each potential, and the averages were plotted.

\section{Microfluidic transport on a gold electrode with micropillars}

Previous experiments have shown that a microscopic three-dimensional structure not only enhances but also accelerates the change caused by electrowetting. The observed phenomenon should also have a positive effect in the transport of solutions in microfluidic devices. First, we used the device shown in Fig. 2(a) ${ }^{13,39}$ and examined the manner in

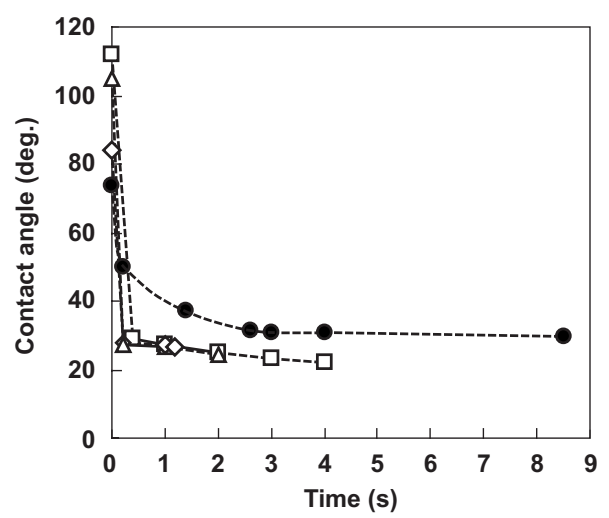

FIG. 6. Changes of the contact angle to a step change of potential from $0 \mathrm{~V}$ to $-1.0 \mathrm{~V}(f=0.45)$. Diameter of micropillars: $\square, 10 \mu \mathrm{m} ; \Delta, 20 \mu \mathrm{m}$; $\diamond$, $50 \mu \mathrm{m}$. shows the contact angle on a smooth electrode. 
(a)

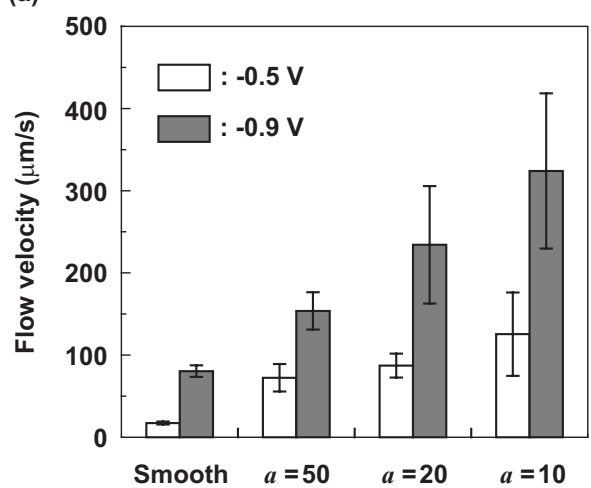

(b)

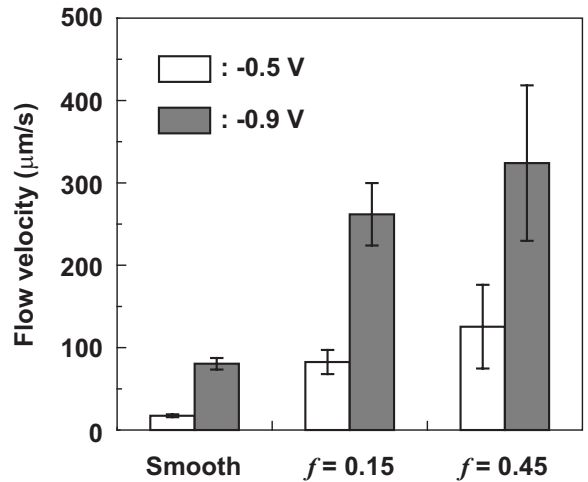

FIG. 7. Flow velocity of a column of a $1.0 \mathrm{M} \mathrm{KCl}$ solution at -0.5 and -0.9 V. (a) Dependence of the flow velocity on $a(\mu \mathrm{m})(f=0.45)$. (b) Dependence of the flow velocity on $f(a=10 \mu \mathrm{m})$. Three runs were made for each experiment, and the averages and standard deviations are shown.

which the transport of a liquid column was accelerated. Figure 7(a) shows the dependence of the flow velocity on the diameter of the micropillars with a fixed $f(0.45)$. The result obtained on a smooth electrode is also shown for comparison. Reflecting the changes observed with a sessile drop, the flow velocity in the flow channel increased with the decrease in the diameter of the micropillars. When a working electrode with micropillars with a $10 \mu \mathrm{m}$ diameter was used, a fourfold increase was observed in the flow velocity compared to that on a smooth electrode. The effect of micropillars was also examined by decreasing the applied potential to $-0.5 \mathrm{~V}$. With micropillars of $a=10 \mu \mathrm{m}$, the flow velocity was about seven times larger than that on a smooth electrode. The effect of changing $f$ was also examined with a fixed $a(10 \mu \mathrm{m})$ [Fig. 7(b)]. As in the case of changing $a$, the flow velocity increased with the increase in $f$. When $f=0.45$, a $24 \%$ increase was observed compared to the case of $f$ $=0.15$. The same tendency was also observed when the potential was $-0.5 \mathrm{~V}$. It should be noted that the flow velocity on the electrode with the smallest micropillars $(a=10 \mu \mathrm{m})$ and/or the larger $f$ was larger than that on the smooth electrode at $-0.9 \mathrm{~V}$. In other words, the applied potential can be reduced to achieve the same velocity as with the smooth electrode.

We also measured the flow velocity using the solutions with different electrolytes [Fig. 8(a)]. The result obtained on a smooth electrode is also shown for comparison. Reflecting the change of the shape of a sessile drop (Fig. 5), the flow (a)

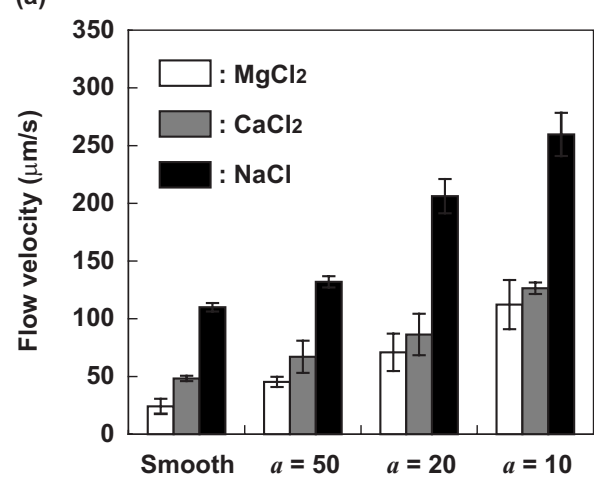

(b)

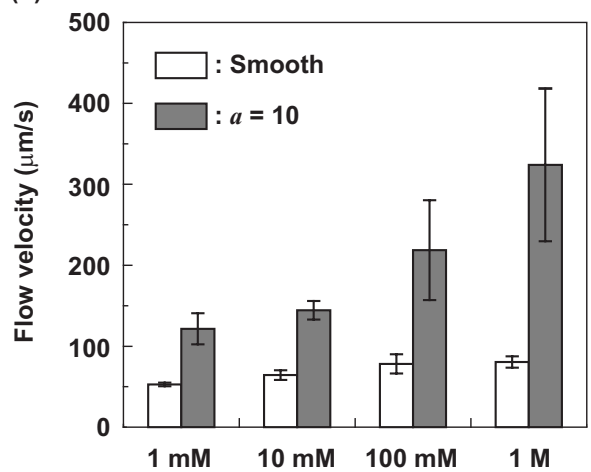

FIG. 8. Influence of the (a) electrolytes and (b) concentration on the flow velocity $(-0.9 \mathrm{~V}, f=0.45)$. (a) Flow velocities measured with solutions of $\mathrm{MgCl}_{2}, \mathrm{CaCl}_{2}$, and $\mathrm{NaCl}$ on a smooth electrode and electrodes with micropillars $(a=50,20,10 \mu \mathrm{m})$. (b) Flow velocities measured with solutions of different concentrations of $\mathrm{KCl}$ on a smooth electrode and electrodes with micropillars $(a=10 \mu \mathrm{m})$. Three runs were made for each experiment, and the averages and standard deviations are shown.

velocity with the $\mathrm{CaCl}_{2}$ or $\mathrm{MgCl}_{2}$ solution was much smaller than that with the $\mathrm{KCl}$ solution, although the flow velocity and its dependence on potential were close between the solutions of divalent cations. Similarly, when the $\mathrm{NaCl}$ solution was used, the flow velocity and its dependence on potential were similar to those with the $\mathrm{KCl}$ solution.

Next, we examined the influence of the electrolyte concentration. Figure 8(b) shows the dependence of the flow velocity on the $\mathrm{KCl}$ concentration. Devices with a smooth electrode or with an electrode with micropillars $(a=10 \mu \mathrm{m}$, $f=0.45$ ) were used. As a general tendency, the flow velocity increased with the increase in the $\mathrm{KCl}$ concentration. However, a remarkable effect was observed with micropillars. On the smooth surface, the flow velocity increased by $53 \%$ when the concentration was increased from $1 \mathrm{mM}$ to $1 \mathrm{M}$. On the other hand, with micropillars, a 2.7-fold increase was observed. In other words, the effect of micropillars is reduced with a decrease in the concentration. In $\mathrm{KCl}$ solutions of different concentrations, the potential of the $\mathrm{Ag} / \mathrm{AgCl}$ reference electrode shifts depending on the concentration of $\mathrm{Cl}^{-}$ ions. The same description applies to the abovementioned experiments using divalent cations. To evaluate this point, the potential shift was corrected, and the same experiment was carried out using the $1 \mathrm{mM}$ solution with which the potential shift was the largest. However, the difference in the flow velocity was less than 5\% compared to the case without 

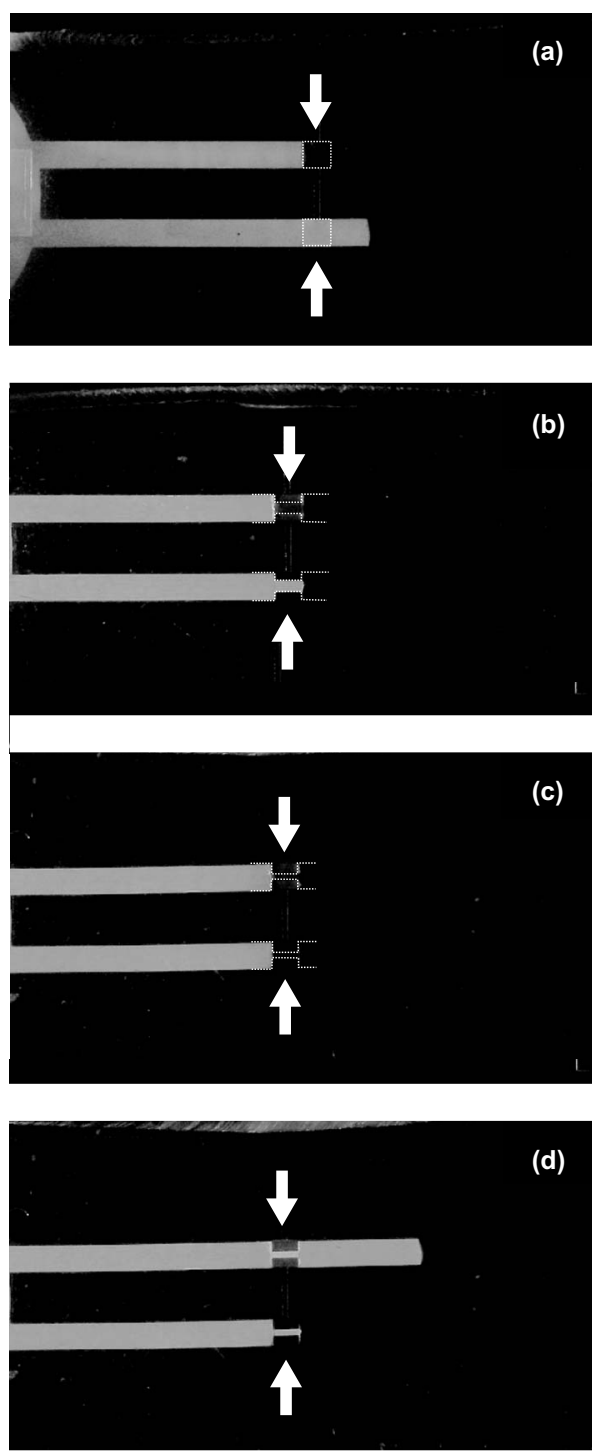

FIG. 9. Movement of a fluorescein solution containing $1.0 \mathrm{M} \mathrm{KCl}$ in the valve area before $[(a)-(c)]$ and after (d) the valves were switched on. Width of the valve area: (a) $500 \mu \mathrm{m}$; (b) $200 \mu \mathrm{m}$; and [(c) and (d)] $100 \mu \mathrm{m}$. The length of the valve area was $500 \mu \mathrm{m}$. The arrows indicate the position of the valves.

correction, which can be explained by the fact that the significant change in wettability occurs in a limited range of potential.

\section{E. Application to a valve}

In the device discussed in the previous section, a potential was applied while a liquid column was mobilized on a long gold electrode. For more efficient microfluidic transport, another option is to mobilize the solution by a capillary action and control the movement using a short strip of electrode that works as a valve [Fig. 2(c)]..$^{40}$ The approach also circumvents a possible adverse influence accompanying electrode reactions. As in the previous long working electrodes, the valve electrode was formed on a micropillar structure with $a=10 \mu \mathrm{m}$ and $f=0.45$.

Figure 9(a) shows the movement of a solution in a straight flow channel with a $500 \mu \mathrm{m}$ width. When a smooth valve electrode was used, the valve action was not satisfac- tory. Although we prepared electrodes whose length ranged from $300 \mu \mathrm{m}$ to $1 \mathrm{~mm}$, the resistive force generated on the hydrophobic PDMS walls could not withstand the motive force generated on the gold electrode. As a result, the solution passed the electrode area uncontrollably. With regard to the stopping ability, the microstructures showed a remarkable effect. For all cases, the solution stopped at the entrance of the valve area. The stopping ability of the valve depends on the width of the valve area and is improved by narrowing the area. ${ }^{40}$ Therefore, we then narrowed only the valve area to 100 or $200 \mu \mathrm{m}$ [Figs. 9(b) and 9(c)]. With the smooth electrode, not even the 200- $\mu$ m-wide valve could stop the solution. However, by reducing the width to $100 \mu \mathrm{m}$, the solution could be stopped at the entrance of the valve area. Indeed, the valves with the microstructures could stop the solution as well as in the previous experiments.

Moreover, we examined the changes after the potential was switched on. Figure 9(d) shows the movements of the liquid columns after the $100-\mu \mathrm{m}$-wide valves with different electrode structures were opened at the same time. When using the electrode with micropillars, the time required for the solution to pass the valve area was $2.5 \mathrm{~s}$ on the average. On the other hand, the liquid column moved more slowly on the smooth electrode, spending $5.9 \mathrm{~s}$ on the average. The difference in the position of the meniscus of the liquid columns is evident in Fig. 9(d). The movement of the solution should increase with a wider flow channel. ${ }^{40}$ When the $500-\mu \mathrm{m}$-wide valve with the micropillars was used, the time required for the solution to pass the valve area was $0.7 \mathrm{~s}$ on the average. According to the obtained results, a wider electrode with a microscopic structure realizes more reliable and faster switching.

\section{CONCLUSIONS}

A microscopic three-dimensional structure enhances the change caused by electrowetting on a gold electrode. The change in the contact angle is enhanced as the diameter of micropillars decreases and/or the fraction of the top area of micropillars with respect to the projected area increases. The smaller changes observed with the solutions of $\mathrm{CaCl}_{2}$ and $\mathrm{MgCl}_{2}$ or the solutions with lower $\mathrm{KCl}$ concentrations can also be enhanced using microscopic three-dimensional structures. Furthermore, the changes observed on the electrodes with micropillars are more rapid than those observed on the smooth electrodes. In a microfluidic device that uses a gold electrode to generate the driving force, the flow velocity increases remarkably by the formation of micropillars, which conform to the behavior of sessile drops. The enhanced changes reduce the applied voltage minimizing adverse influences, including the electrolysis of water. A valve formed with an electrode with micropillars can stop the intruding solution more effectively than a valve with a smooth electrode. Furthermore, the switching speed can be improved with electrodes with micropillars.

\section{ACKNOWLEDGMENTS}

This study was supported by a Grant-in-Aid for Scientific Research on Priority Areas (Grant No. 17040008) under 
the Ministry of Education, Culture, Sports, Science, and Technology, Japan.

${ }^{1}$ C. Quilliet and B. Berge, Curr. Opin. Colloid Interface Sci. 6, 34 (2001) ${ }^{2}$ F. Mugele and J.-C. Baret, J. Phys.: Condens. Matter 17, R705 (2005).

${ }^{3}$ F. Sassa, K. Morimoto, W. Satoh, and H. Suzuki, Electrophoresis 29, 1787 (2008).

${ }^{4}$ V. Srinivasan, V. K. Pamula, and R. B. Fair, Lab Chip 4, 310 (2004).

${ }^{5}$ M. G. Pollack, R. B. Fair, and A. D. Shenderov, Appl. Phys. Lett. 77, 1725 (2000).

${ }^{6}$ J. Lee, H. Moon, J. Fowler, T. Schoellhammer, and C.-J. Kim, Sens. Actuators, A 95, 259 (2002).

${ }^{7}$ M. G. Pollack, A. D. Shenderov, and R. B. Fair, Lab Chip 2, 96 (2002).

${ }^{8}$ H. Moon, S. K. Cho, R. L. Garrell, and C.-J. Kim, J. Appl. Phys. 92, 4080 (2002).

${ }^{9}$ P. Paik, V. K. Pamula, and R. B. Fair, Lab Chip 3, 253 (2003).

${ }^{10}$ P. Y. Chiou, H. Moon, H. Toshiyoshi, C. J. Kim, and M. C. Wu, Sens. Actuators, A 104, 222 (2003).

${ }^{11}$ V. Srinivasan, V. K. Pamula, and R. B. Fair, Anal. Chim. Acta 507, 145 (2004).

${ }^{12}$ I. Moon and J. Kim, Sens. Actuators, A 130-131, 537 (2006).

${ }^{13}$ W. Satoh, H. Hosono, and H. Suzuki, Anal. Chem. 77, 6857 (2005).

${ }^{14}$ N. Nashida, W. Satoh, J. Fukuda, and H. Suzuki, Biosens. Bioelectron. 22 3167 (2007).

${ }^{15}$ H. Hosono, W. Satoh, M. Toya, K. Morimoto, J. Fukuda, and H. Suzuki, Sens. Actuators B 132, 614 (2008)

${ }^{16}$ T. Onda, S. Shibuichi, N. Satoh, and K. Tsujii, Langmuir 12, 2125 (1996).

${ }^{17}$ H. Y. Erbil, A. L. Demirel, Y. Avci, and O. Mert, Science 299, 1377 (2003).

${ }^{18}$ K. Tadanaga, K. Kitamuro, J. Morinaga, Y. Kotani, A. Matsuda, and T. Minami, Chem. Lett. 29, 864 (2000).

${ }^{19}$ K. Teshima, H. Sugimura, Y. Inoue, O. Takai, and A. Takano, Appl. Surf.
Sci. 244, 619 (2005).

${ }^{20}$ M. Li, J. Zhai, H. Liu, Y. Song, L. Jiang, and D. Zhu, J. Phys. Chem. 107, 9954 (2003)

${ }^{21}$ M. E. Abdelsalam, P. N. Bartlett, T. Kelf, and J. Baumberg, Langmuir 21, 1753 (2005)

${ }^{22}$ W. Sun, S. Zhou, P. Chen, and L. Peng, Chem. Commun. (Cambridge) 2008, 603.

${ }^{23}$ M. Hashimoto, N. Sakamoto, S. Upadhyay, J. Fukuda, and H. Suzuki, Biosens. Bioelectron. 22, 3154 (2007)

${ }^{24}$ A. Hozumi and O. Takai, Thin Solid Films 303, 222 (1997).

${ }^{25}$ P. Favia, G. Cicala, A. Milella, F. Palumbo, P. Rossini, and R. d'Agostino, Surf. Coat. Technol. 169-170, 609 (2003).

${ }^{26}$ J. Bico, C. Marzolin, and D. Quéré, Europhys. Lett. 47, 220 (1999).

${ }^{27}$ B. He, N. A. Patankar, and J. Lee, Langmuir 19, 4999 (2003).

${ }^{28}$ N. J. Shirtcliffe, S. Aqil, C. Evans, G. McHale, M. I. Newton, C. C. Perry, and P. Roach, J. Micromech. Microeng. 14, 1384 (2004).

${ }^{29}$ J. Jopp, H. Grüll, and R. Yerushalmi-Rozen, Langmuir 20, 10015 (2004).

${ }^{30}$ T. N. Krupenkin, J. A. Taylor, T. M. Schneider, and S. Yang, Langmuir 20, 3824 (2004).

${ }^{31}$ J. O. M. Bockris, A. K. N. Reddy, and M. Gamboa-Aldeco, Modern Electrochemistry (Kluwer, Dordrecht/Plenum, New York, 2000), Vol. 2A, Chap. 6.

${ }^{32}$ R. N. Wenzel, Ind. Eng. Chem. 28, 988 (1936).

${ }^{33}$ A. B. D. Cassie and S. Baxter, Trans. Faraday Soc. 40, 546 (1944).

${ }^{34}$ H. Suzuki and T. Taura, J. Electrochem. Soc. 148, E468 (2001).

${ }^{35}$ T. Smith, J. Colloid Interface Sci. 75, 51 (1980).

${ }^{36}$ L. Chai and J. Klein, Langmuir 23, 7777 (2007).

${ }^{37}$ W. Pan, C. J. Durning, and N. J. Turro, Langmuir 12, 4469 (1996).

${ }^{38}$ S. Shibuichi, T. Onda, N. Satoh, and K. Tsujii, J. Phys. Chem. 100, 19512 (1996).

${ }^{39}$ W. Satoh, M. Loughran, and H. Suzuki, J. Appl. Phys. 96, 835 (2004).

${ }^{40}$ W. Satoh, H. Yokomaku, H. Hosono, N. Ohnishi, and H. Suzuki, J. Appl. Phys. 103, 034903 (2008). 